\title{
Neoplasias hematológicas: un mundo aún por descubrir
}

Hematologic neoplasms: a world yet to be discovered

La Hematología es esa "ciencia roja» llena de células de diferentes tamaños, colores y sin sabores, porque, ya lo confirmarán algunos colegas, que amargo y doloroso es encontrar una célula neoplásica en el extendido de sangre periférica de un paciente. Pero realmente ¿estamos conscientes los profesionales que trabajamos en esta área clínica de la presencia de estas células? y ¿por qué el temor de tantos, el respeto de algunos y la indiferencia de otros por conocer, estudiar y poner ojos y corazón en esa estructura observada a través del microscopio? De allí la importancia del compromiso y la dedicación en nuestra labor al servicio de la comunidad.

Entrando entonces a ese aún enigmático mundo de las neoplasias hematológicas podemos encontrar que existe, según la clasificación de la Organización Mundial de la Salud (OMS), una enorme variedad y que, a partir del descubrimiento de diferentes alteraciones genéticas y moleculares se está avanzando para alcanzar la predicción del comportamiento clínico, pronóstico y el tratamiento de estas entidades. El esquema de clasificación de la OMS parte primero del linaje celular maligno: linfoide, mieloide, histiocítico/dendrítico y mastocítico, y los diferencia de acuerdo con una mezcla interesante de las características morfológicas, el inmunofenotipo, la genética, la biología molecular y los síndromes clínicos relacionados.

La nueva clasificación de la OMS presentada este año (2016), elaborada gracias al consenso entre hemato-oncólogos, patólogos, hematólogos y genetistas, incluye los cambios de las neoplasias hematológicas actuales, como la adición de un número limitado de nuevas entidades provisionales, esclareciendo el diagnóstico y tratamiento en las primeras etapas; además, refina los criterios diagnósticos, moleculares, la citogenética y su correlación clínica.

En la actualidad, algunas neoplasias hematológicas aún son incurables, una de ellas es el mieloma múltiple, una enfermedad maligna, sistémica, que resulta de la proliferación clonal de células plasmáticas derivadas de los linfocitos $B$, en la cual hay producción anómala de una proteína $M$ en el suero, la orina o ambas. Esta entidad es la neoplasia hematológica más frecuente después del linfoma no Hodgkin y, según la OMS, representa el $1 \%$ de todas las neoplasias y el $10 \%$ al $15 \%$ de las neoplasias hematológicas.

Desde una mirada holística, el impacto del mieloma múltiple en la salud es relativamente bajo si se le compara con condiciones patológicas más frecuentes como las enfermedades cardiovasculares; sin embargo, la incapacidad generada en los pacientes en edad productiva y el elevado costo del tratamiento denota un impacto importante sobre la economía/salud, principalmente si se trata de un país en vía de desarrollo como el nuestro. Cabe señalar que, a pesar de ser considerado una enfermedad de la vejez, está siendo descrito en pacientes menores de 40 años, incluso menores de 30 años; esto debido a los avances diagnósticos y tecnológicos. En Colombia esta situación es más compleja y la principal causa es nuestro sistema de salud, además de la baja sospecha clínica, lo cual limita el diagnóstico y, por consiguiente, el tratamiento. Se hace entonces demandante el hecho de realizar un diagnóstico oportuno, ya que, 
según diversas investigaciones, se puede prolongar la expectativa de vida de los pacientes, previniendo el daño a órganos e incluso las complicaciones derivadas, que constituyen las principales causas de muerte, estando en primer lugar las infecciones.

Al otro lado de este panorama se encuentra otra entidad hematológica que, en este caso, afecta principalmente a la base de nuestra sociedad, los niños, y representa la primera causa de muerte por enfermedad en esta población. Se trata de las leucemias agudas, principalmente la leucemia linfoblástica aguda, las cuales se pueden originar en células del linaje linfoide o mieloide en diferentes etapas de madurez en la médula ósea. En la literatura dicen que la leucemia en niños es una enfermedad poco común, pero entonces ¿por qué muchos de nosotros la vemos a diario en la práctica clínica en esta población? Es una realidad terrible, que a su vez, es un aliciente para que los Bacteriólogos y Microbiólogos, desde nuestra profesión y humanidad, aportemos al diagnóstico desde la base que es la visualización correcta de los blastos en sangre periférica, que permita y exija a los Médicos instaurar el tratamiento correcto y oportuno.

La incidencia de las neoplasias hematológicas en niños es mayor en los países desarrollados; sin embargo, en los países subdesarrollados puede estar subestimada. Aún hay muchos datos que se desconocen sobre la epidemiología del cáncer en el niño, por lo que son necesarios más estudios. Según el Protocolo de Vigilancia en Salud Pública de las leucemias agudas pediátricas, Colombia presenta tasas altas de incidencia (seis casos nuevos por 100.000 niños y 5,6 casos nuevos por 100.000 niñas), lo que sugiere que el diagnóstico definitivo y el tratamiento presentan demoras y tasas de abandono que contribuyen a una alta mortalidad ( $45 \%$ de las defunciones por cáncer en menores de 15 años), a lo que se le suma el hecho de que no se cuentan con los beneficios de avances terapéuticos que sí tienen otros países.

Por tanto, las neoplasias hematológicas, al día de hoy, siguen constituyendo un cúmulo de conocimientos aún por descubrir, describir e investigar.

En este número de Medicina \& Laboratorio, en el módulo "La Clínica y el Laboratorio», se incluye una revisión sobre las leucemias mieloides agudas en niños, la segunda neoplasia hematológica más frecuente en este grupo de población, titulada «Enfoque diagnóstico de las leucemias mieloides agudas pediátricas», en la que se hace un abordaje sobre este tema desde el punto de vista de la clasificación y el abordaje diagnóstico desde el laboratorio, con énfasis en la población pediátrica.

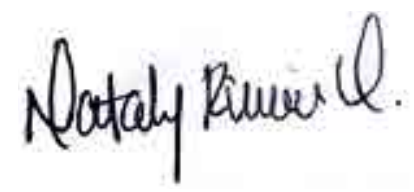

Nataly J. Rincón Vásquez. MB.

Coordinadora área Hematología, Hospital General de Medellín

Medellín, Colombia, abril 2016 